\title{
Association between the use of antibiotics during pregnancy and obesity in 5-year-old children
}

\author{
Chunyu Zhuang ${ }^{1 \#}$, Xueli Liao ${ }^{2 \#}$, Mianai Fu ${ }^{3}$, Huiling Shi ${ }^{4}$ Bailang Lin ${ }^{1}$, Canyang Zhu ${ }^{2}$, Qiuxia Chen ${ }^{5}$, \\ Biwei Mai ${ }^{6}$, Rui Liu ${ }^{4}$ \\ ${ }^{1}$ Department of Nursing, Haikou Hospital of the Maternal and Child Health, Haikou, China; ${ }^{2}$ Department of Pharmaceutical, Hainan Women and \\ Children's Medical Center, Haikou, China; ${ }^{3}$ Reproductive Medicine Centre, Haikou Hospital of the Maternal and Child Health, Haikou, China; \\ ${ }^{4}$ Child Healthcare Department, Haikou Hospital of the Maternal and Child Health, Haikou, China; ${ }^{5}$ Department of Obstetrics, Hainan Women and \\ Children's Medical Center, Haikou, China; ${ }^{6}$ Department of Pediatrics, Hainan Women and Children's Medical Center, Haikou, China \\ Contributions: (I) Conception and design: C Zhuang, X Liao, R Liu; (II) Administrative support: M Fu, H Shi, B Lin; (III) Provision of study materials \\ or patients: C Zhu, Q Chen, B Mai; (IV) Collection and assembly of data: All authors; (V) Data analysis and interpretation: C Zhuang, X Liao, R Liu; \\ (VI) Manuscript writing: All authors; (VII) Final approval of manuscript: All authors. \\ \#These authors contributed equally to this work. \\ Correspondence to: Rui Liu. Child Healthcare Department, Haikou Hospital of the Maternal and Child Health, No. 6 Wentan Road, Guoxing Avenue, \\ Haikou 570203, China. Email: liurui3953@163.com.
}

Background: Obesity is an epidemic that must arouse our attention. The purpose of this research is to investigate the relationship between antibiotic use during pregnancy and childhood obesity in 5-year-old Chinese children.

Methods: A total of 132 5-year-old children born in our hospital from January 2014 to January 2016 were included. The children's genders, nationalities, chronic diseases, and other factors were collected as adjusted covariates. We also collected the pre-pregnancy body mass indexes (BMIs) of pregnant women, diagnosis of diabetes mellitus or gestational diabetes mellitus (GDM), maternal smoking, mode of delivery (cesarean section and vaginal delivery), and weight gain during pregnancy, which may affect childhood obesity. A linear mixed effect regression model was used to test the correlation between antibiotic use during pregnancy and the BMI of 5-year-old children, and a logistic mixed effect regression model was used to evaluate the correlation between antibiotic use during pregnancy and obesity outcome.

Results: When adjusted for the child's gender, birth weight, cesarean section, chronic diseases, maternal smoking during pregnancy, pre-pregnancy BMI, diagnosis of diabetes or GDM, mode of delivery (cesarean section and vaginal delivery), and weight gain during pregnancy (Model 3), antibiotic use during pregnancy was positively correlated with the BMI of 5-year-old children ( $\beta=0.01,95 \% \mathrm{CI}$ : $0.01-0.03, \mathrm{P}=0.04)$. Antibiotic use during pregnancy increased the risk of obesity by $4 \%$ in 5 -year-old children $(\mathrm{OR}=1.04,95 \%$ CI: $1.01-1.16, \mathrm{P}=0.02)$.

Conclusions: The use of antibiotics during pregnancy is positively correlated with the risk of obesity in 5-year-old children.

Keywords: Antibiotics; pregnancy; obesity; children

Submitted Apr 23, 2021. Accepted for publication Jun 11, 2021.

doi: $10.21037 /$ tp-21-220

View this article at: https://dx.doi.org/10.21037/tp-21-220 


\section{Introduction}

Obesity is an important public health problem affecting $33.7 \%$ of adults and $14.0 \%$ of children worldwide, which requires an urgent solution $(1,2)$. Previous studies have found that several factors in the fetal period play a decisive role in obesity (3). For example, pre-pregnancy body mass index (BMI), weight gain during pregnancy, smoking, and cesarean section delivery are independently correlated with the increased risk of childhood obesity (4-8).

Recent studies have identified potential effects of early antibiotic exposure in childhood on the metabolism and growth of children (9). The relationship between the use of antibiotics during pregnancy and childhood obesity has also received attention and discussion. In a retrospective cohort study by Popovic et al. (9), a higher risk of childhood obesity at 3 years of age was observed if the mother took antibiotics during pregnancy. However, this was only because antibiotic use during pregnancy was associated with antibiotic use earlier in childhood (10). Moreover, a mediating effect of maternal weight on childhood obesity was revealed. In another cohort study of Danish schoolchildren, Mueller et al. reported a higher adjusted prevalence of obesity among children aged 7-16 years with prenatal exposure to antibiotics (11).

Given that the conclusions of previous studies exhibited a certain variability, and most of them were conducted in European and American populations resulting in a certain population bias, the present study aims to explore the correlation between the use of antibiotics during pregnancy and childhood obesity in 5-year-old Chinese children. We present the following article in accordance with the STROBE reporting checklist (available at https://dx.doi. org/10.21037/tp-21-220).

\section{Methods}

\section{Objects}

A total of 1325 -year-old children born in our hospital from January 2014 to January 2016 were enrolled in our study. The inclusion criteria were as follows: (I) availability of complete birth records; (II) availability of complete medication records of the mother during pregnancy as well as relevant information during pregnancy; (III) children aged 5 years or above; and (IV) complete records of the children's height and weight at 5 years of age. All procedures performed in this study involving human participants were in accordance with the Declaration of Helsinki (as revised in 2013). The study was approved by the Ethics Committee of Haikou Hospital of the Maternal and Child Health (No.: 2013HN063) and informed consent was taken from all the patients.

\section{Data collection}

Information on the use of antibiotics by the mother during pregnancy were collected. Antibiotic use during pregnancy was defined as an outpatient prescription of antibiotics by a pregnant woman at 9 months (273 days) before the child's birth. At the same time, according to the dosage of antibiotics, antibiotic use was divided into 0-4+, narrow spectrum antibiotics (amoxicillin, penicillin, dicloxacillin, etc.), and broad-spectrum antibiotics (such as the amoxicillin/clavulanate group).

The obesity status in 5-year-old children was the primary outcome of this study. We defined childhood obesity as age- and sex-specific BMI $\geq$ that of $85 \%$ of the population. Gender, ethnicity, chronic disease, and other factors of children were collected as the covariates of correction. We also collected data on maternal pre-pregnancy BMI, diagnosis of diabetes or gestational diabetes mellitus (GDM), maternal smoking, mode of delivery (cesarean section $v s$. vaginal delivery), and weight gain during pregnancy, which may contribute to childhood obesity.

\section{Statistical analysis}

SAS 9.4 software (North Carolina State University, USA) was used for statistical analysis. Categorical variables were presented as the number of cases (percentage), and continuous variables were presented as the mean \pm standard deviation or median (interquartile interval). We used a linear mixed effect regression model to examine the association between antibiotic use during pregnancy and BMI in 5-year-old children, and a logistic mixed effect regression model to evaluate the association between antibiotic use during pregnancy and obesity outcomes.

\section{Results}

\section{Basic data of the objects}

A total of 132 children were enrolled. Of these, girls accounted for $51.5 \%$, and the mean birth weight was $3.3 \pm 0.6 \mathrm{~kg}$. Caesarean section accounted for $9.8 \%$; those with chronic diseases accounted for $3.0 \%$; $4.5 \%$ of the children's 
mothers smoked during pregnancy; $45.4 \%$ of mothers had a BMI $\geq 25 \mathrm{~kg} / \mathrm{m}^{2}$ before pregnancy; $22.0 \%$ of mothers had diabetes or GDM; and the proportion of antibiotic use during pregnancy was $25.8 \%$, as shown in Table 1 .

\section{Correlation between the use of antibiotics during pregnancy and the BMI of 5-year-old children}

Multiple linear regression analysis indicated that antibiotic use during pregnancy was positively associated with BMI at

Table 1 Basic characteristics of the study objects

\begin{tabular}{lc}
\hline Characteristics & Number $(\mathrm{N}=132)$ \\
\hline Mothers & $6(4.5)$ \\
Smoking during pregnancy $(\mathrm{n}, \%)$ & $62(45.4)$ \\
BMl before pregnancy $\geq 25 \mathrm{~kg} / \mathrm{m}^{2}(\mathrm{n}, \%)$ & $12.7 \pm 3.1$ \\
Weight gain during pregnancy $(\mathrm{kg})$ & $29(22.0)$ \\
Diabetes mellitus or gestational diabetes & \\
mellitus (n, \%) & $34(25.8)$ \\
Antibiotic use during pregnancy $(\mathrm{n}, \%)$ & $28(21.2)$ \\
0 & $2(1.5)$ \\
1 & $2(1.5)$ \\
2 & $1(0.8)$ \\
3 & $1(0.8)$ \\
$4+$ & $14(10.6)$ \\
Narrow-spectrum antibiotics $(\mathrm{n}, \%)$ & $20(21.2)$ \\
Broad-spectrum antibiotics $(\mathrm{n}, \%)$ & \\
Children & $68(51.5)$ \\
Girl (n, \%) & $3.3 \pm 0.6$ \\
Birth weight (kg) & $13(9.8)$ \\
Caesarean section $(\mathrm{n}, \%)$ & $4(3.0)$ \\
\hline Chronic disease (n, \%) & \\
\hline
\end{tabular}

BMI, body mass index.
5 years of age $[\beta=0.03,95 \%$ confidence interval (CI): $0.02-$ $0.06, \mathrm{P}=0.02]$ without any adjustment (Model 1 ). Even after adjustment for the child's gender, birth weight, cesarean section delivery, and chronic disease (Model 2), this positive association persisted ( $\beta=0.02,95 \% \mathrm{CI}$ : 0.01-0.04, $\mathrm{P}=0.03$ ). After further adjustment for maternal smoking during pregnancy, pre-pregnancy BMI, diagnosis of diabetes mellitus or GDM, method of delivery (cesarean section $v$ s. vaginal delivery), and gestational weight gain (Model 3), this positive association remained ( $\beta=0.01,95 \%$ CI: $0.01-0.03$, $\mathrm{P}=0.04$ ) as shown in Table 2.

\section{Correlation between antibiotic use during pregnancy and obesity in 5-year-old children}

Logistic regression analysis demonstrated that when unadjusted (Model 1), antibiotic use during pregnancy increased the risk of obesity by $17 \%$ in 5 -year-old children (OR $=1.17,95 \% \mathrm{CI}: 1.11-1.36, \mathrm{P}=0.003)$. When adjusted for the child's gender, birth weight, cesarean section, and chronic disease (Model 2), antibiotic use during pregnancy increased the risk of obesity by $14 \%$ in 5 -year-old children $(\mathrm{OR}=1.14,95 \% \mathrm{CI}: 1.10-1.31, \mathrm{P}=0.007)$. When further adjusted for maternal smoking during pregnancy, prepregnancy BMI, diagnosis of diabetes or GDM, method of delivery (cesarean section $v s$. vaginal delivery), and gestational weight gain (Model 3), antibiotic use during pregnancy increased the risk of obesity by $4 \%$ in 5 -year-old children (OR $=1.04,95 \% \mathrm{CI}$ : $1.01-1.16, \mathrm{P}=0.02)$, as shown in Table 3.

\section{Subgroup analysis of the association between antibiotic use during pregnancy and obesity in 5-year-old children}

Adequately adjusted for the child's gender, birth weight, cesarean section, chronic disease, maternal smoking during pregnancy, pre-pregnancy BMI, diabetes or GDM diagnosis, delivery method (cesarean section $v s$. vaginal delivery), and gestational weight gain, stratified analysis

Table 2 Correlation between the use of antibiotics during pregnancy and BMI of 5-year-old children

\begin{tabular}{lccc}
\hline Models & $\beta$ & $95 \%$ Cl & P value \\
\hline Model 1 & 0.03 & $0.02-0.06$ & 0.02 \\
Model 2 & 0.02 & $0.01-0.04$ & 0.03 \\
Model 3 & 0.01 & $0.01-0.03$ & 0.04 \\
\hline
\end{tabular}

BMI, body mass index. 
Table 3 Correlation between antibiotic use during pregnancy and obesity in 5-year-old children

\begin{tabular}{lccc}
\hline Models & OR & $95 \% \mathrm{Cl}$ & $\mathrm{P}$ value \\
\hline Model 1 & 1.17 & $1.11-1.36$ & 0.003 \\
Model 2 & 1.14 & $1.10-1.31$ & 0.007 \\
Model 3 & 1.04 & $1.01-1.16$ & 0.02 \\
\hline
\end{tabular}

Table 4 Subgroup analysis of the association between antibiotic use during pregnancy and obesity in 5-year-old children

\begin{tabular}{lccc}
\hline Variables & OR & $95 \% \mathrm{Cl}$ & $\mathrm{P}$ value \\
\hline Antibiotic dose & & & $0.01-1.32$ \\
1 & 1.01 & $1.01-1.03$ & 0.03 \\
2 & 1.02 & $1.01-1.05$ & 0.02 \\
3 & 1.03 & $1.02-1.08$ & 0.01 \\
$4+$ & 1.05 & & 0.14 \\
Types of antibiotics & & $0.02-1.29$ & 0.001 \\
Narrow-spectrum antibiotics & 1.16 & $1.13-1.42$ & 0.21 \\
Broad-spectrum antibiotics & 1.21 & \\
\hline
\end{tabular}

(by antibiotic dose and type) found that there was no significant association between an antibiotic dose (level 1) and the risk of obesity in 5 -year-old children $(\mathrm{OR}=1.01$, 95\% CI: 0.01-1.32, $\mathrm{P}=0.06$ ); an antibiotic dose (level 2) was associated with a $2 \%$ increased risk of obesity at age 5 (OR $=1.02,95 \%$ CI: $1.01-1.03, \mathrm{P}=0.03$ ); an antibiotic dose (level 3 ) increased the risk of obesity at age 5 by $3 \%(\mathrm{OR}=1.03$, 95\% CI: $1.01-1.05, \mathrm{P}=0.02$ ); and an antibiotic dose (level of $4+$ ) was associated with a $5 \%$ increased risk of obesity at age $5(\mathrm{OR}=1.05,95 \% \mathrm{CI}: 1.02-1.08, \mathrm{P}=0.01)$. There was no significant association between narrow-spectrum antibiotics and the risk of obesity in 5-year-old children (OR $=1.16,95 \%$ CI: $0.02-1.29, \mathrm{P}=0.14)$, while broad-spectrum antibiotics increased the risk of obesity by $21 \%$ at age 5 (OR $=1.21,95 \%$ CI: $1.13-1.42, \mathrm{P}=0.001)$, as shown in Table 4 .

\section{Discussion}

The present study demonstrated a significant positive association between antibiotic use during pregnancy and the risk of obesity in children aged 5 years. After fully adjusting the possible influencing factors of both the mother and child on the obesity of 5-year-old children, this study still found that the risk of obesity in 5 -year-old children increased with higher doses of antibiotics used during pregnancy.
Furthermore, the use of broad-spectrum antibiotics was more significantly associated with an increased risk of obesity in 5 -year-old children.

Consistent with previous studies, we also observed a potential association between maternal antibiotics during pregnancy and later childhood obesity. A study by Mor et al. of 436 mothers and infants showed that antibiotics exposure in the second and third trimesters of pregnancy increased the risk of obesity by $84 \%$ in children aged 7 years (12). It has also been reported that the use of antibiotics by mothers during pregnancy is significantly positively correlated with the risk of childhood obesity (OR $=1.29,95 \%$ CI: $1.03-1.62$, $\mathrm{P}=0.01$ ) (13). A recent study of 303 mothers and infants showed that exposure to antibiotics during pregnancy resulted in a small increase in infants' BMI during followup 2 years after birth (14).

Mothers often need to receive antibiotic treatment during pregnancy, and the most common indication is urinary tract infection, which is often quickly and effectively treated (15). Research into the potential mechanism of changes in the intestinal microbiome or epigenetic programming (16-18) suggests that the use of antibiotics during pregnancy may have an innate influence on the intestinal microbiome of children, thereby mediating the occurrence of obesity in later childhood. This may explain 
why the use of broad-spectrum antibiotics was associated with a greater risk of obesity at 5 years of age compared to narrow-spectrum antibiotics in the present study, as broadspectrum antibiotics have a wider antibacterial spectrum and have a greater impact on the child's intestinal flora. In addition, it has been found that neonatal blood with mothers infected during pregnancy is rich in antibodies (19). These children have been born with strong innate immunity and are not prone to stunting due to low immunity in childhood.

In this study, the use of antibiotics during pregnancy was found to be correlated with obesity in 5-year-old children, which may be helpful to better analyze the rising incidence and pathogenesis of obesity in children. Additionally, our study discovered this correlation in the Chinese population for the first time, which carries a certain suggestive effect on the use of antibiotics during pregnancy in the future. Considering the risk of using antibiotics during pregnancy for offspring's obesity, pregnant women should be instructed to avoid using antibiotics as much as possible during pregnancy. For patients who have already used antibiotics, the intestinal flora of the offspring should be closely monitored after birth, probiotics should be supplemented in a planned way, physical exercise should be strengthened, and the intestinal flora should be checked regularly. However, this study also has some limitations that should be noted. Firstly, the use of antibiotics included in this study was established using only the prescription records of outpatients. The use of antibiotics by pregnant women in out-of-hospital pharmacies or other hospitals was not fully understood, which may mean that our analysis is biased to some extent. Secondly, the sample size of this study was small, and thus, the research conclusions need to be verified in future studies with a larger sample sizes.

In conclusion, the present study identified a significant positive association between antibiotic use during pregnancy and the risk of obesity in 5 -year-old children.

\section{Acknowledgments}

Funding: None.

\section{Footnote}

Reporting Checklist: The authors have completed the STROBE reporting checklist. Available at https://dx.doi. org/10.21037/tp-21-220
Data Sharing Statement: Available at https://dx.doi. org/10.21037/tp-21-220

Conflicts of Interest: All authors have completed the ICMJE uniform disclosure form (available at https://dx.doi. org/10.21037/tp-21-220). The authors have no conflicts of interest to declare.

Etbical Statement: The authors are accountable for all aspects of the work in ensuring that questions related to the accuracy or integrity of any part of the work are appropriately investigated and resolved. All procedures performed in this study involving human participants were in accordance with the Declaration of Helsinki (as revised in 2013). The study was approved by the Ethics Committee of Haikou Hospital of the Maternal and Child Health (No.: 2013HN063) and informed consent was taken from all the patients.

Open Access Statement: This is an Open Access article distributed in accordance with the Creative Commons Attribution-NonCommercial-NoDerivs 4.0 International License (CC BY-NC-ND 4.0), which permits the noncommercial replication and distribution of the article with the strict proviso that no changes or edits are made and the original work is properly cited (including links to both the formal publication through the relevant DOI and the license). See: https://creativecommons.org/licenses/by-nc-nd/4.0/.

\section{References}

1. NCD Risk Factor Collaboration (NCD-RisC). Worldwide trends in body-mass index, underweight, overweight, and obesity from 1975 to 2016: a pooled analysis of 2416 population-based measurement studies in 128.9 million children, adolescents, and adults. Lancet 2017;390:2627-42.

2. Commission on Ending Childhood Obesity. Report of the Commission on Ending Childhood Obesity. World Health Organization, Geneva; 2016.

3. Gluckman PD, Hanson MA, Cooper C, et al. Effect of in utero and early-life conditions on adult health and disease. N Engl J Med 2008;359:61-73.

4. LifeCycle Project-Maternal Obesity and Childhood Outcomes Study Group, Voerman E, Santos S, et al. Association of Gestational Weight Gain With Adverse Maternal and Infant Outcomes. JAMA 2019;321:1702-15.

5. Woo Baidal JA, Locks LM, Cheng ER, et al. Risk 
Factors for Childhood Obesity in the First 1,000 Days: A Systematic Review. Am J Prev Med 2016;50:761-79.

6. Stiemsma LT, Michels KB. The Role of the Microbiome in the Developmental Origins of Health and Disease. Pediatrics 2018;141:e20172437.

7. Palmsten K, Hernández-Díaz S, Chambers CD, et al. The Most Commonly Dispensed Prescription Medications Among Pregnant Women Enrolled in the U.S. Medicaid Program. Obstet Gynecol 2015;126:465-73.

8. Stephansson O, Granath F, Svensson T, et al. Drug use during pregnancy in Sweden - assessed by the Prescribed Drug Register and the Medical Birth Register. Clin Epidemiol 2011;3:43-50.

9. Popovic M, Rusconi F, Zugna D, et al. Prenatal exposure to antibiotics and wheezing in infancy: a birth cohort study. Eur Respir J 2016;47:810-7.

10. Cox LM, Blaser MJ. Antibiotics in early life and obesity. Nat Rev Endocrinol 2015;11:182-90.

11. Mueller NT, Whyatt R, Hoepner L, et al. Prenatal exposure to antibiotics, cesarean section and risk of childhood obesity. Int J Obes (Lond) 2015;39:665-70.

12. Mor A, Antonsen S, Kahlert J, et al. Prenatal exposure to systemic antibacterials and overweight and obesity in Danish schoolchildren: a prevalence study. Int J Obes (Lond) 2015;39:1450-5.

Cite this article as: Zhuang $\mathrm{C}$, Liao $\mathrm{X}, \mathrm{Fu} \mathrm{M}$, Shi H, Lin B, Zhu C, Chen Q, Mai B, Liu R. Association between the use of antibiotics during pregnancy and obesity in 5-year-old children. Transl Pediatr 2021;10(6):1686-1691. doi: 10.21037/tp-21-220
13. Poulsen MN, Pollak J, Bailey-Davis L, et al. Associations of prenatal and childhood antibiotic use with child body mass index at age 3 years. Obesity (Silver Spring) 2017;25:438-44.

14. Cassidy-Bushrow AE, Burmeister C, Havstad S, et al. Prenatal antimicrobial use and early-childhood body mass index. Int J Obes (Lond) 2018;42:1-7.

15. Wang B, Liu J, Zhang Y, et al. Prenatal Exposure to Antibiotics and Risk of Childhood Obesity in a Multicenter Cohort Study. Am J Epidemiol 2018;187:2159-67.

16. Heerman WJ, Daley MF, Boone-Heinonen J, et al. Maternal antibiotic use during pregnancy and childhood obesity at age 5 years. Int J Obes (Lond) 2019;43:1202-9.

17. Jess T, Morgen CS, Harpsøe MC, et al. Antibiotic use during pregnancy and childhood overweight: A populationbased nationwide cohort study. Sci Rep 2019;9:11528.

18. Brown RG, Al-Memar M, Marchesi JR, et al. Establishment of vaginal microbiota composition in early pregnancy and its association with subsequent preterm prelabor rupture of the fetal membranes. Transl Res 2019;207:30-43.

19. Fettweis JM, Serrano MG, Brooks JP, et al. The vaginal microbiome and preterm birth. Nat Med 2019;25:1012-21.

(English Language Editor: A. Kassem) 